\title{
UTILIZAÇÃO DE TÉCNICAS DE OTIMIZAÇÃO NO DIMENSIONAMENTO DE VIGAS DE CONCRETO ARMADO
}

\author{
M. B. LEITE ${ }^{1}$ e W. M. PEREIRA JUNIOR ${ }^{2}$ \\ Universidade Federal de Goiás \\ matheus.b.leite@outlook.com ${ }^{1}$
}

Artigo submetido em 13/09/2017 e aceito em 04/04/2019

DOI: $10.15628 /$ holos.2019.6282

\section{RESUMO}

Com um mercado cada vez mais competitivo e empresas buscando a livre concorrência, uma preocupação que está sempre presente é a redução de custos. Essa preocupação, atualmente, também está relacionada com o meio ambiente, sendo necessário pensar em uma redução do uso dos recursos naturais. Dessa forma, sistemas estruturais devem ser projetados para que expectativas como essas possam ser atendidas, desde que respeitem os critérios estabelecidos pelas normas de dimensionamento vigentes. O presente trabalho tem como foco aplicar conceitos de otimização no dimensionamento de vigas de concreto armado em seção retangular. O presente trabalho apresenta uma revisão bibliográfica sobre o dimensionamento de vigas como também os conceitos gerais de otimização. Portanto propõe-se a aplicação de do método clássico de Newton com penalidade exterior para a minimização de uma função objetivo (FO) de custo. A FO é restrita pelos critérios de dimensionamento informados na NBR 6118 (ABNT, 2014). Assim foi verificado a validade da rotina de cálculo proposta (WJM_otimiza) e também verificou-se a aplicação da mesma em problemas reais. Para os casos analisados o custo da viga variou entre $\mathrm{R} \$ 86,63$ a $\mathrm{R} \$$ 453,24 .

PALAVRASCHAVE: Otimização, Vigas de concreto armado, Penalidade Exterior (MPE), Método de Newton.

\section{USE OF OPTIMIZATION TECHNIQUES IN THE DESIGN OF REINFORCED CONCRETE BEAMS}

\begin{abstract}
Due an increasingly competitive market and companies seeking for free competition, a concern always present is cost reduction. This concern, nowadays, involves the environmental as well, and it is necessary to think about a reduction in the use of natural resources. Thus, structural systems must be designed so expectations such these can be satisfied, as long as they follow design criteria of building standards. The present paper aims to apply the concepts of optimization in the design of reinforced concrete beams with rectangular section. It also introduces a literature review of beam design and general concepts of optimization.
\end{abstract}

Therefore, it is proposed the application of classic Newton method with exterior penalty to minimize the objective function (OF) of costs. The function is described by design criteria found in NBR 6118 (ABNT, 2014). Thus, it was verified the validation of calculation routine proposed (WJM_otimiza) and it was also verified the application of that routine in real problems. For the analysed cases, the beam cost varied between $\mathrm{R} \$ 86,64$ to $\mathrm{R} \$ 453,24$.

KEYWORDS: Optimization, Reinforced Concrete beams, Exterior penalty, Newton method. 


\section{INTRODUÇÃO}

A otimização é a parte da ciência que procura encontrar, de forma sistematizada, a melhor solução para um determinado problema, atendendo às condições que limitam o espaço das soluções viáveis, denominadas restrições do problema, segundo um critério que mede a qualidade de cada solução, denominado função - objetivo. As técnicas de otimização direcionam uma busca sistematizada da solução ótima, onde o processo, além de ser executado de forma automatizada, fornece a melhor solução do problema, sem que seja necessário pesquisar todas as possíveis soluções (VASCONCELOS, 2014) por processo de tentativa e erro. Portanto do ponto de vista projetual, a aplicação das técnicas de otimização torna-se uma ferramenta interessante, visto que as mesmas podem reduzir o tempo de elaboração de um projeto (VASCONCELOS et al., 2013).

Assim sendo, é essencial que em projetos de estruturas, o engenheiro investigue técnicas para otimizar o dimensionamento das seções transversais de vigas, uma vez que as mesmas têm um papel fundamental no suporte de carregamentos em geral de uma estrutura (LIU et al., 2007), (MUÑIZ JUNIOR e OLIVEIRA, 2014). Klein (2008) também reafirma a necessidade de que todos os projetos envolvidos na grande área das engenharias passem por um processo de otimização. Sendo assim, atende-se uma premissa essencial para os engenheiros: (a) Economia e (b) bom desempenho.

Vanderplaats (2006) e Maia (2009) afirmam que o uso de conceitos de otimização estrutural se deu a partir da década de 60 com os estudos de Schmidt, e desde então, tal ferramenta viabilizou o desenvolvimento e projeto de novos produtos. São três os processos possíveis para otimizar um elemento estrutural qualquer, sendo eles: (a) Otimização de seção; (b) Otimização de forma; (c) Otimização de topologia. Neste artigo, a problematização desenrola-se entorno do dimensionamento da seção transversal de viga de concreto armado, portanto, será aplicado um algoritmo para otimização da seção.

Alguns métodos de otimização vêm sendo utilizados para o dimensionamento de elementos em geral, como por exemplo o trabalho de Klein (2008), que utilizou o método do Algoritmo Genético (AG) e o método do Gradiente Reduzido Generalizado (GRG) para otimizações de seções de vigas retangulares de concreto armado. Muñiz Junior e Oliveira (2014) utilizou a função solver do Excel como ferramenta para minimização do custo de uma viga de concreto armado. Dessa forma o autor obteve ábacos "altura ótima versus vão" para peças de concreto armado Classe C25 e C50. Já Braun (2016) utiliza técnicas de otimização para minimizar o custo de vigas de aço em seção I, através do algoritmo de otimização Harmony Search.

Leps e Sejnohga (2003) aplica o algoritmo de recozimento simulado para determinar o menor custo possível para vigas em concreto armado biapoiadas, baseado nos critérios de dimensionamento do EUROCODE 2. Os critérios restritivos da função foram para flexão simples e cisalhamento.

Dessa forma, devido à importância do conceito de otimização no âmbito de projetos de engenharia, o presente artigo tem como objetivo: (a)Aplicar o método clássico de Newton para 
minimizar uma função de custo de vigas de seção retangular em concreto armado submetido à flexão; (b) Validar a implementação de um algoritmo de otimização para um problema de análise e dimensionamento estrutural; e (c) Encontrar as variáveis de projeto (no caso altura e área de aço) que minimizam uma função de custo de uma viga biapoiada).

Este artigo está organizado da seguinte forma: Na Seção 2 são apresentados os conceitos teóricos a respeito de otimização, como também os aspectos da NBR 6118 (ABNT, 2014) sobre dimensionamento de vigas submetidas a flexão. Já a seção 3 demonstra como serão realizados os testes numéricos com as vigas de concreto, os dados iniciais, além da formulação do problema de otimização para este artigo. A seção 4 aborda os resultados do artigo e a seção 5 conclui o mesmo.

\section{REVISÃO BIBLIOGRÁFICA}

A seguir serão apresentados os conceitos teóricos necessários para o desenvolvimento deste trabalho. Primeiramente, uma abordagem sobre os métodos de otimização, e, em seguida, a dedução do problema de flexão em vigas de concreto armado, sendo que esse último recorre a uma abordagem normativa de dimensionamento.

\subsection{Otimização e seus conceitos básicos}

Segundo Vanderplaats (1999), um problema geral de otimização pode ser definido através de uma Função Objetivo (FO), as variáveis de projeto, as restrições do problema e o espaço viável de busca do projeto. Portando a formulação geral pode ser descrita pelas Equações (1) a (5)

Minimizar $f\left(x_{1}, x_{2}, \ldots x_{n}\right)$

Restrições de igualdade:

$h\left(x_{1}, x_{2}, . . x_{n}\right)=0$

Restrições de desigualdade:

$$
g\left(x_{1}, x_{2}, . . x_{n}\right) \leq 0
$$

Restrições laterais:

$$
X_{i} \leq X \leq X_{j}
$$

Variáveis de projeto 


$$
X=\left\{\begin{array}{l}
x_{1} \\
x_{2} \\
\cdots \\
x_{n}
\end{array}\right\}
$$

Portanto, o objetivo de um processo de otimização é encontrar os valores do vetor $\{X\}$ que minimizam a FO do problema. Para isso utiliza-se um processo iterativo para delimitar a solução ótima (Ver Equação 6) do problema, sendo que a direção de busca é o que diferencia os métodos de otimização em geral, alterando, para cada um deles, a variável $S$.

$$
X^{k+1}=X^{k}+\alpha \cdot S\left(X^{k}\right)
$$

Onde $X^{k+1}$ é a variável de projeto atualizada no passo $k+1, \alpha$ é o passo de cada iteração e $S\left(X^{k}\right)$ é a direção de busca no ponto $X^{k}$.

\subsection{Métodos clássicos de otimização}

De acordo com (SILVA, 20--) e Vanderplaats (1999), os métodos de otimização podem ser classificados como de ordem zero, primeira ordem e de segunda ordem. Os métodos de ordem zero não utilizam informações das derivadas da função, como por exemplo, o método da seção áurea, sequência de Fibonacci, método de Powell, etc. Nos métodos de primeira ordem, são utilizadas as informações da primeira derivada da FO para determinar a direção de busca do problema, destacando-se o método da máxima descida e o método da descida conjugada. Os métodos de segunda ordem são os que utilizam as informações da segunda derivada para a direção de busca, destacando-se o método de Newton.

Abaixo serão detalhados os métodos de otimização utilizados neste artigo, são eles: (a) Método da Seção Áurea; e (b) Método de Newton. Para consideração das restrições será apresentado e utilizado o Método da Penalidade Exterior Quadrática (MPE).

\subsubsection{Método unidimensional da seção áurea}

A relação áurea ou número de ouro $(\Phi)$ é definido pelo número algébrico irracional $(1+$ $\sqrt{5}) / 2$ que é exatamente a solução da equação quadrática $x^{2}-x-1=0$. A relação áurea fascinou intelectuais de diversas áreas durante 24 séculos, visto que, se associa a diversos fenômenos físicos notáveis (SEM \& AGARWAL, 2008) como também em diversas partes da natureza. Abbas (2017) afirma que o número de ouro é uma espécie de proporção perfeita ou número perfeito matematicamente. Nos manuscritos do Homem Vitruviano (1490) elaborados por Leonardo da Vinci (1452-1519) as proporções do corpo humano são equivalentes ao número de ouro.

Em relação a seção áurea como método de otimização, o mesmo se caracteriza por ser um método de ordem zero, e, normalmente, é utilizado para determinar o valor do passo (ver Equação 6) a cada iteração do processo de otimização. $O$ algoritmo calcula o ponto ótimo de uma função quase convexa unidimensional, em uma determinada região (Ver Figura 1a), dentro de um intervalo $a$ e $b \in R$ (chamado intervalo de incerteza). O procedimento da seção áurea consiste em 
uma redução sucessiva de intervalos que devem manter simetria em relação aos limites $a$ e $b$ (HERNÁNDEZ et al., 2012). Em linhas gerais, a cada iteração o processo elimina um trecho do intervalo correspondente a constante $(1-\rho) \cong 0,618033989$. O processo iterativo de busca do novo intervalo é dado pelas Equações (7) e (8):

$$
\begin{aligned}
& a_{k}=a_{k+1}+\rho\left(b_{k+1}-a_{k+1}\right) \\
& b_{k}=a_{k+1}+(1-\rho) \cdot\left(b_{k+1}-a_{k+1}\right)
\end{aligned}
$$

Se $f\left(a_{1}\right)<f\left(b_{1}\right)$, o ponto mínimo $x^{*}$ da função deve estar no intervalo $\left[a_{0}, b_{1}\right]$ e para situações onde $f\left(a_{1}\right)>f\left(b_{1}\right)$, o ponto o mínimo $x^{*}$ deve estar no intervalo $\left[a_{1}, b_{0}\right]$ (Ver Figura 1b).

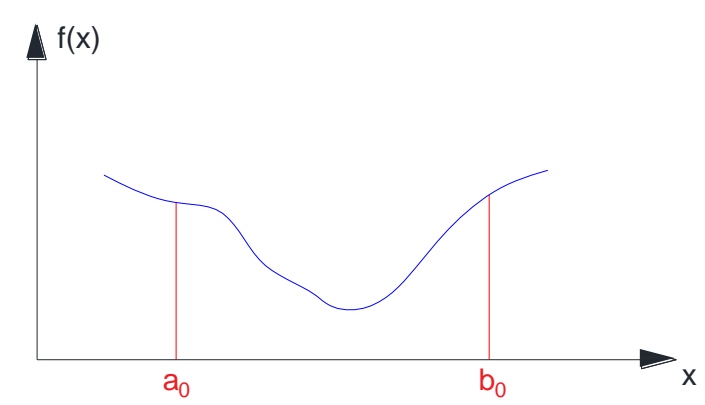

(a)

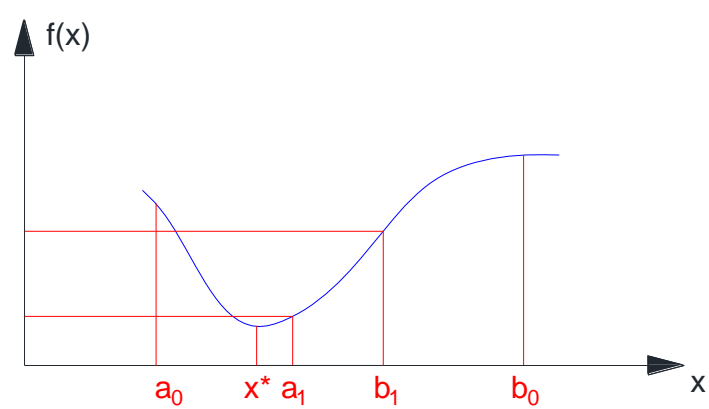

(b)

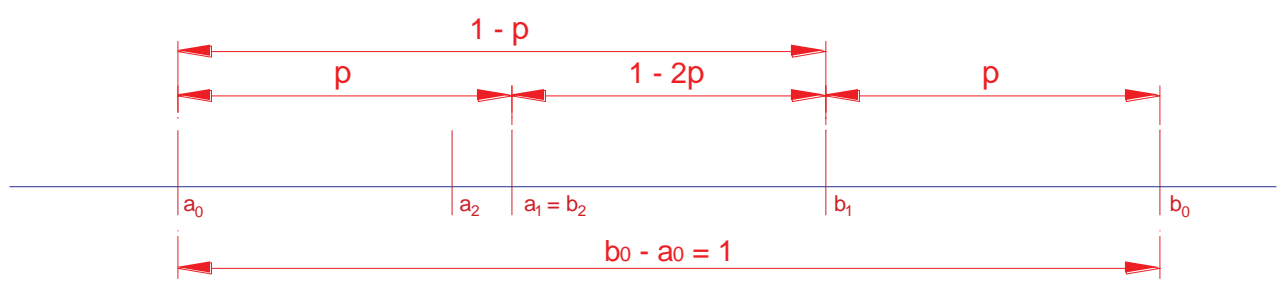

(c)

Figura 1: Análise gráfica do método da seção áurea (a) Intervalo de incerteza da função unidimensional; (b) Amostra do processo iterativo após uma iteração; (c) Verificação dos intervalos de incerteza da função.

A simetria dos intervalos é garantida pela Equação (9):

$$
a_{1}-a_{0}=b_{1}-b_{0}=\rho\left(b_{0}-a_{0}\right)
$$

Para um número $n$ de iterações o intervalo sofrerá uma redução igual à Equação (10).

$$
(1-\rho)^{n} \cong 0,61803^{n}
$$

O método da seção áurea tem sua aplicação acoplada a outros métodos de otimização e em problemas mais robustos o mesmo é utilizado para calcular o valor do passo $(\alpha)$ conforme Equação (6).

\subsubsection{Método irrestrito de Newton}

Como citado anteriormente, o método de Newton é um método de 2o ordem, pois, depende das informações contidas na segunda derivada da FO. Para problemas multidimensionais, essa informação é adquirida através da matriz Hessiana da FO. Do ponto de vista do processo geral 
de otimização apresentado na seção 2.1, as informações da segunda derivada são armazenadas na direção de busca $S\left(X^{k}\right)$ da Equação (6).

Uma das desvantagens do método é a necessidade de se avaliar a matriz Hessiana da FO, dado que esse processo tem um alto custo computacional para problemas fortemente não lineares, e que também é preciso garantir que a segunda derivada da FO seja continua. Dessa forma, tem-se que a direção de busca pelo método Newton é dado pela Equação (11).

$$
S\left(X^{k}\right)=-H\left(X^{k}\right)^{-1} \cdot \nabla f\left(X^{k}\right)
$$

Onde $H\left(X^{k}\right)$ é a matriz Hessiana no ponto $X^{k}$ e $\nabla f\left(X^{k}\right)$ é a matriz Hessiana no ponto $X^{k}$. No caso em questão, o método de Newton será utilizado para a solução de uma função irrestrita, função essa que será obtida após aplicação do método da penalidade exterior (MPE) que será apresentado a seguir.

\subsubsection{Métodos de penalidade para otimização restrita}

Os métodos de penalidade para otimização restrita baseiam-se em modelos que criam uma função pseudo-objetivo $(\Phi)$, em que a FO é corrigida de acordo com as restrições do problema, permitindo que o mesmo seja resolvido de forma clássica e irrestrita. A formulação geral da pseudo-objetivo é dada pela Equação (12).

$$
\phi\left(X^{k}, r p\right)=F O\left(X^{k}\right)+r p . P\left(X^{k}\right)
$$

Onde $F O$ é a função objetivo do problema, $r p$ é o multiplicador iterativo de penalidade e $P\left(X^{k}\right)$ são as funções de penalidade.

Basicamente são três os métodos de penalidade para aplicação a funções restritas, são eles: (a) Método da Penalidade Exterior (MPE); (b) Método da Penalidade Interior (MPI); e (c) Método de Lagrange Aumentado (VANDERPLAATS, 1999). Para este artigo foi adotado o método MPE.

\subsubsection{Método da Penalidade Exterior (MPE)}

Os métodos de penalidade exterior são utilizados em problemas que envolvem funções com restrição de igualdade e de desigualdade (MOTA, 2010); (VENKATARAMAN, 2001); e (VANDERPLAATS, 1999). Dessa forma, esses podem ser usados em problemas como o do dimensionamento de uma viga submetida à flexão.

Para o método da penalidade exterior, a função de penalidade é dada pela Equação (13):

$$
P\left(X^{k}\right)=\sum_{j=1}^{m}\left\{\max \left[0, g_{j}\left(X^{k}\right)\right]\right\}^{2}+\sum_{k=1}^{n}\left[h_{k}\left(X^{k}\right)\right]^{2}
$$

Onde $g_{j}\left(X^{k}\right)$ são as restrições de desigualdade e $h_{k}\left(X^{k}\right)$ são as restrições de igualdade do problema analisado, e, para garantir que a tangente à função de penalidade seja nula sobre a fronteira do espaço viável, as restrições são elevadas ao quadrado, assegurando-se assim, a continuidade da derivada da função pseudo-objetivo. 
Ao longo do processo iterativo é provocada uma penalização na FO de forma que as variáveis de projeto se desloquem da região não viável para a fronteira da região viável de projeto (MOTA, 2010). Desse modo, quando o ponto mínimo se encontra dentro do espaço viável, tem-se que $P\left(X^{k}\right)=0$. Percebe-se, então, que a função pseudo-objetivo é igual a função objetivo.

\subsection{Dimensionamento à flexão simples em vigas de concreto}

Segundo Bastos (2015), vigas com armaduras simples seriam aquelas com seção que necessitam apenas de uma armadura longitudinal resistente tracionada, apesar de ter armadura na seção comprimida - somente por técnicas construtivas - sendo considerado para cálculo somente a resistência do concreto para as tensões de compressão solicitadas.

A NBR 6118 (ABNT, 2014) permite o dimensionamento apenas no domínio 2 e em partes do domínio 3, respeitando os limites de $x / d$ impostos pela mesma. Esses limites são dados pelas Equações (14) e (15).

$$
\begin{aligned}
& x / d \leq 0,35, \text { para } f_{c k}>50 \mathrm{MPa} \\
& x / d \leq 0,45, \text { para } f_{c k} \leq 50 \mathrm{MPa}
\end{aligned}
$$

Onde $x$ é a posição da linha neutra medida a partir do banzo comprimido da seção; $d$ é a altura útil da peça.

\subsubsection{Equacionamento da flexão para armadura simples}

O problema de uma seção de concreto armado sujeita à flexão simples é dada através da Figura 2:

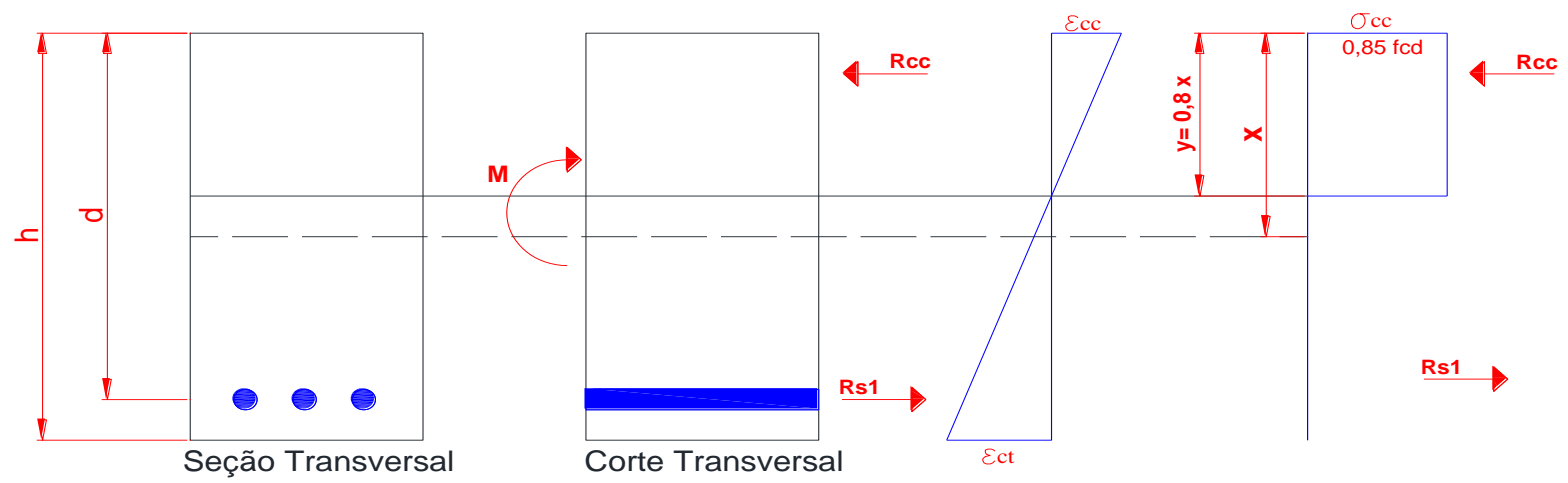

Figura 2: Distribuição de tensões e deformações em viga de seção retangular com armadura simples de acordo com as hipóteses da NBR 6118 (ABNT, 2014)

Para que o equilíbrio da seção seja atendido, as forças horizontais no sistema devem ser iguais a zero e o somatório de momentos igual ao momento de cálculo da seção. Portanto, para o cálculo de vigas com armadura simples, têm-se as Equações (16) a (20).

$$
\begin{aligned}
& R_{c c}=R_{s t} \\
& M_{d}=R_{c c} \cdot z
\end{aligned}
$$




$$
M_{d}=R_{s t} \cdot z
$$

Onde $R_{c c}$ corresponde à força resultante das tensões de compressão, $R_{s t}$ a força resultante das tensões de tração no aço e $M_{d}$ o momento interno gerado (pelo concreto comprimido e armadura tracionada). As Equações (19) e (20) representam os seus equacionamentos.

$$
\begin{aligned}
& R_{c c}=\left(0,85 \cdot f_{c d}\right) \cdot\left(b_{w}\right) \cdot 0,8 \\
& z=d-0,4
\end{aligned}
$$

Onde $f_{c d}\left(f_{c d}=f c k / 1,40\right)$ representa a tensão resistente de compressão de cálculo, $b_{w}$ a largura da viga e $z$ é o braço de alavanca.

Portanto, substituindo a Equação (19) e (20) em (17), chega-se à Equação (21) do Momento de resistente de cálculo $(M d)$ da peça.

$$
M_{d}=\left(0,68 \cdot x \cdot d-0,272 \cdot x^{2}\right) \cdot b_{w} \cdot f_{c d}
$$

Onde $x$ é a posição da linha neutra e $d$ a altura útil da viga. Dessa maneira, pode-se verificar a profundidade da linha neutra pela Equação (22):

$$
x=\frac{0,68 \cdot d \pm\left[(0,68 \cdot d)^{2}-4 \cdot 0,272 \cdot\left(\frac{M_{d}}{b_{w} \cdot f_{c d}}\right)\right]^{\frac{1}{2}}}{0,544}
$$

Com o valor de $x$ calculado é possível encontrar a área de aço necessária para a flexão, de modo a suportar um momento externo $M$ (Ver Figura 2).

$A_{s}=\frac{M_{d}}{z \cdot f_{y d}}$

Onde $A_{s}$ representa a área de aço, $M_{d}$ o momento de cálculo e $f_{y d}$ a resistência de cálculo do aço e o valor de fyd é dado por: $f_{y d}=f_{y k} / 1,15$

Com a consideração das deformações lineares, pode-se estabelecer a Equação (24) que apresenta as deformações em função da linha neutra da peça.

$$
\frac{\varepsilon_{c d}}{\varepsilon_{s d}}=\frac{x}{d-x}
$$

Onde, $\varepsilon_{c d}$ corresponde à deformação específica do concreto comprimido e $\varepsilon_{s d}$ é a deformação específica da armadura tracionada.

\section{MATERIAIS E MÉTODOS}

Nessa seção são apresentados os materiais e métodos empregados neste trabalho, de forma a atingir os objetivos do mesmo. Tal artigo se enquadra em uma pesquisa exploratória e 
aplicada, pois utiliza-se de dados numéricos para fazer as análises do custo de uma estrutura de viga real, explorando a temática de aplicação da otimização em projetos estruturais.

\subsection{Procedimento de modelagem}

O problema analisado trata-se do dimensionamento à flexão de seções retangulares de concreto armado. Nesse caso, foi avaliado um problema de minimização de uma função de custo, sendo que as variáveis de projeto são altura a viga ( $x 1)$ e a área de aço (x2).

Para obtenção dos valores ótimos da variável de projeto, utilizou-se o método da penalidade exterior (MPE) combinado ao método de $2 \stackrel{0}{\circ}$ ordem de Newton.

Foi elaborado um algoritmo denominado WJM_otimiza, que acopla os métodos irrestritos e restritos, tal fluxograma está descrito a seguir, conforme Figura 3a e $3 \mathrm{~b}$. O mesmo se divide em uma rotina de macrofluxo que diz respeito ao código principal do programa, que para o caso deste trabalho, trata-se do método restrito de Penalidade Exterior (MPE) e um microfluxo que diz respeito ao funcionamento do método irrestrito (Método de Newton), ambos necessários para o completo funcionamento dessa aplicação.

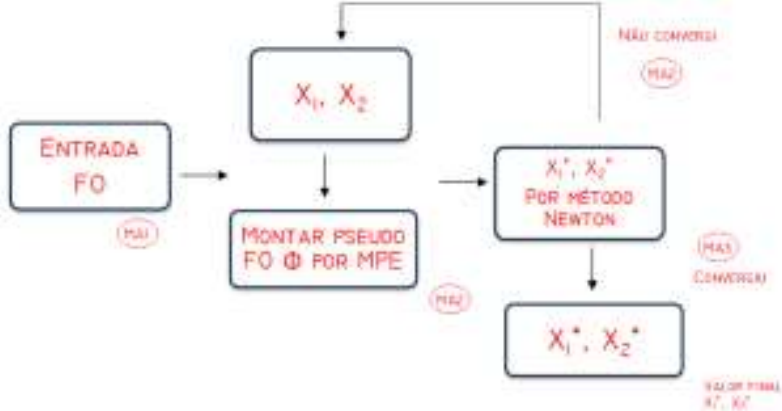

(a) Macrofluxo da rotina para contemplar as restrições e montar a Pseudo FO

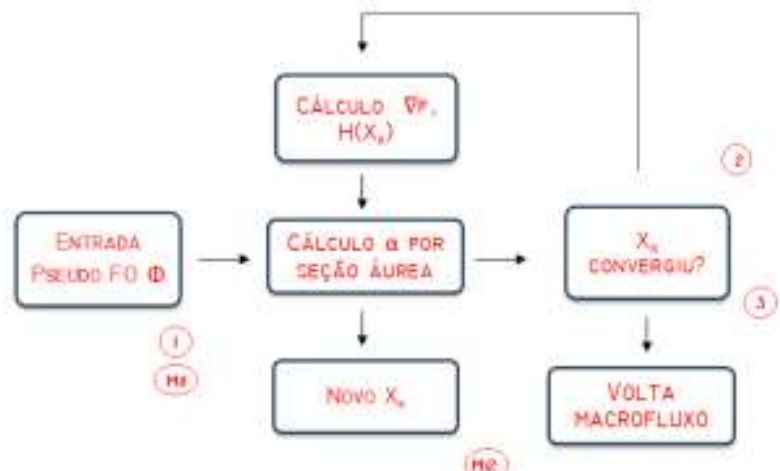

(b) Microfluxo da rotina para resolver o problema irrestrito

Figura 3: Macrofluxo e Microfluxo rotina WJM_otimiza referente a parte restrita.

Para realização das análises, aplicou-se o problema de minimização de custo com restrições baseadas no dimensionamento à flexão simples de seções retangulares de concreto armado. Já o modelo de viga utilizado está descrito na Figura 4.
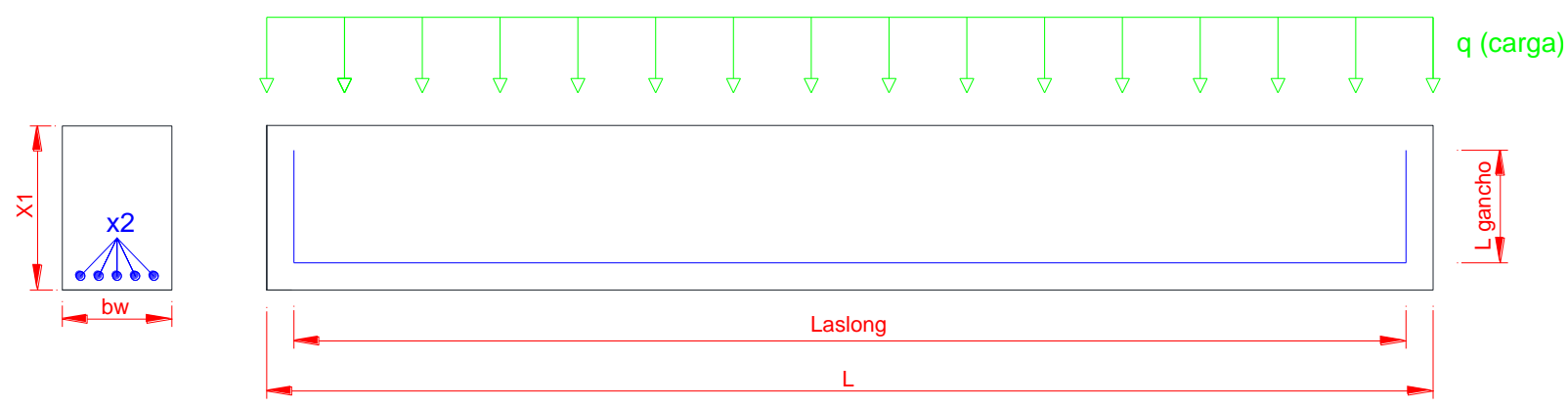

Figura 4: Modelo da viga que foi utilizada no método de otimização.

O custo total de produção de uma viga é dado através do somatório dos custos dos materiais constituintes, que são: Custo de forma (CF); Custo de concreto (CC); Custo de aço (CA). 
Portanto a FO utilizada para esse problema é descrita nas Equações (25) a (31) apresentadas a seguir. Tal equacionamento é fundamentado no texto de Klein (2008).

$$
C t=C F+C C+C A
$$

Onde:

$$
\begin{aligned}
& C F=(2 \cdot x 1+b w) \cdot L \cdot \frac{P C M}{10^{4}} \\
& C C=x 1 \cdot b w \cdot L \cdot \frac{P C V}{10^{6}} \\
& C A=x 2 \cdot L_{\text {total }} \cdot \rho \cdot P \\
& L_{\text {total }}=L_{\text {aslong }}+L_{\text {gancho }} \\
& L_{\text {aslong }}=L-2 \cdot C N \\
& L_{\text {gancho }}=(x 1-2 \cdot C N) .2
\end{aligned}
$$

Foi adotado um cobrimento nominal $C N=2,50 \mathrm{~cm}$ e a densidade do aço $\rho=7850 \mathrm{~kg} / \mathrm{m}^{3}$, sendo $P C M$ o preço da chapa de madeira por $\mathrm{m}^{2}, P C V$ o preço do volume de concreto por $\mathrm{m}^{3}$ e $P A$ é o preço do aço por $\mathrm{kg}$.

Para definição dos valores dos materiais, foi utilizada a base de dados da Caixa Econômica Federal (CEF) chamada Sistema Nacional de Pesquisa de Custos e Índices da Construção Civil (SINAPI). Para tal levantamento de custo, foi utilizado o mês de Novembro de 2016 como referência. Foram escolhidas composições desoneradas, e, o valor de cada serviço é dado conforme Quadro 1 apresentado logo a seguir.

Quadro 1: Valor monetário dos materiais utilizados na definição da FO.

\begin{tabular}{|l|c|c|}
\hline \multicolumn{1}{|c|}{ Material } & \multicolumn{1}{|}{$\begin{array}{c}\text { Unidade de } \\
\text { medida }\end{array}$} & Preço (R\$) \\
\hline Chapa de madeira para forma (PCM) & $\mathrm{m}^{2}$ & 31,93 \\
\hline Concreto usinado (PCV) & $\mathrm{m}^{3}$ & 296,22 \\
\hline Vergalhão de aço CA-50 (PA) & $\mathrm{kg}$ & 4,44 \\
\hline
\end{tabular}

De acordo com as informações do SINAPI, os materiais analisados têm as seguintes características: (a) Chapa de madeira para fôrma com espessura de $18 \mathrm{~mm}$; (b) Concreto usinado bombeável, C30, incluindo custo com bombeamento; (c) Aço CA-50, vergalhão de bitola de $10 \mathrm{~mm}$ de diâmetro.

Após completa manipulação algébrica e substituição de valores, a FO completa é dada pela Equação (32).

$$
C T=(2 \cdot x 1+b w) \cdot L \cdot \frac{31,93}{10^{4}}+x 1 \cdot b w \cdot L \cdot \frac{296,22}{10^{6}}+x 2 \cdot[(L-5)+(2 x 1-10)] \frac{7850}{10^{6}} \cdot 4,44
$$

As restrições que foram utilizadas são de igualdade e desigualdade, respeitando as recomendações da NBR 6118 (ABNT, 2014) para dimensionamento. As restrições de desigualdade 
estão relacionadas com a altura e a área de aço, máximas e mínimas. As inequações (33) a (36) apresentam as restrições utilizadas.

$$
\begin{aligned}
& x 1 \geq 30 \mathrm{~cm} \\
& x 1 \leq 60 \mathrm{~cm} \\
& x 2 \geq b w \cdot x 1 \cdot 0,0015 \\
& x 2 \leq b w \cdot x 1 \cdot 0,04
\end{aligned}
$$

As restrições de igualdade estão relacionadas com as equações de equilíbrio de forças e momento fletor. As mesmas são apresentadas nas Equações (37) e (38) a seguir:

$$
\begin{aligned}
& \left(0,85 \cdot f_{c d}\right) \cdot\left(b_{w}\right) \cdot 0,8-\sigma_{s d} \cdot x 2=0 \\
& M_{d}-\left(0,68 \cdot 0,45 \cdot(x 1-2 \cdot C N) \cdot d-0,272 \cdot 0,45 \cdot(x 1-2 \cdot C N)^{2}\right) \cdot b_{w} \cdot f_{c d}=0
\end{aligned}
$$

\subsection{Plano de Análise e Interpretação de Dados}

Antes da realização dos exemplos, foram feitos testes de funcionamento com o algoritmo implementado. Tal validação utilizou o texto de Klein (2008) como base para comparação com os dados obtidos neste artigo. Foi utilizada uma viga de concreto armado, biapoiada, seção retangular, bw $=15 \mathrm{~cm}$, armadura simples, vão livre de 6 metros, submetida a um carregamento (q) distribuído de $10 \mathrm{kN} / \mathrm{m}$ e $20 \mathrm{kN} / \mathrm{m}$ e fck de $25 \mathrm{MPa}$.

Para as análises paramétricas, foi elaborado um conjunto de dados de entrada com

\begin{tabular}{|c|c|c|c|c|c|c|c|}
\hline Simulações & $\begin{array}{c}\mathbf{f}_{\mathrm{ck}} \\
(\mathrm{MPa})\end{array}$ & $\begin{array}{c}f_{y k} \\
(\mathrm{MPa})\end{array}$ & $\begin{array}{l}b_{w} \\
(\mathrm{~cm})\end{array}$ & $\begin{array}{c}\text { Carga } \\
\text { distribuída } q \\
(\mathrm{kN} / \mathrm{m}) \\
\end{array}$ & $\mathrm{L}(\mathrm{cm})$ & $\begin{array}{l}x 1 \text { inicial } \\
(\mathrm{cm})\end{array}$ & $\begin{array}{c}x 2 \text { inicial } \\
\left(\mathrm{cm}^{2}\right)\end{array}$ \\
\hline 1 & 25,00 & 500,00 & 14,00 & $\begin{array}{c}2,5,10,12,15 \\
\text { e } 20\end{array}$ & 400,00 & 45,00 & 0,70 \\
\hline 2 & 25,00 & 500,00 & 14,00 & $\begin{array}{c}2,5,10,12,15 \\
\text { e } 20\end{array}$ & 450,00 & 45,00 & 0,70 \\
\hline 3 & 25,00 & 500,00 & 14,00 & $\begin{array}{c}2,5,10,12,15 \\
\text { e } 20\end{array}$ & 500,00 & 45,00 & 0,70 \\
\hline 4 & 25,00 & 500,00 & 14,00 & $\begin{array}{c}2,5,10,12,15 \\
\text { e } 20\end{array}$ & 550,00 & 45,00 & 0,70 \\
\hline
\end{tabular}
diferentes vãos e carregamentos para o elemento estrutural de viga apresentado na Figura 6 . 0 ponto de partida ou chute inicial para as variáveis $x 1$ e $x 2$ também são descritas na Tabela 1 e a tolerância admitida como critério de parada do método de otimização foi de $10^{-4}$ conforme recomendação de Klein (2008).

Tabela 1: Dados de entrada do problema de otimização para análise estrutural da viga. 


\section{RESULTADOS E DISCUSSÕES}

Nesta seção são apresentados os resultados e suas análises, respectivamente. As interpretações estão divididas em duas partes. A primeira é relativa à validação da função objetivo do problema, já a segunda é relativa às aplicações de acordo com o plano de análise descrito no item 3.2.

\subsection{Validação da função objetivo e comparações}

De forma a validar o algoritmo utilizado nessa pesquisa, apresenta-se os resultados obtidos na comparação do software WJM_otimiza com a técnica heurística apresentada em Klein (2008). O Quadro 2 apresenta tais valores.

\begin{tabular}{|c|c|c|c|c|}
\multicolumn{2}{c|}{ Quadro 2: Validação da função objetivo comparando com uma função semelhante } \\
\hline \multirow{2}{*}{ Métodos } & $\begin{array}{c}\text { Carga distribuída } \\
\mathbf{q}(\mathbf{k N} / \mathbf{m})\end{array}$ & Altura $\mathbf{( c m )}$ & Área de aço $\left.\mathbf{( c m}^{\mathbf{2}}\right)$ & Custo total \\
\hline \multirow{2}{*}{ Klein (2008) } & $10 \mathrm{kN} / \mathrm{m}$ & 35,00 & 5,70 & $\mathrm{R} \$ 462,60$ \\
\cline { 2 - 5 } & $20 \mathrm{kN} / \mathrm{m}$ & 47,00 & 8,30 & $\mathrm{R} \$ 613,60$ \\
\hline \multirow{2}{*}{ WJM_otimiza } & $10 \mathrm{kN} / \mathrm{m}$ & 35,00 & 5,70 & $\mathrm{R} \$ 488,16$ \\
\cline { 2 - 5 } & $20 \mathrm{kN} / \mathrm{m}$ & 47,00 & 8,30 & $\mathrm{R} \$ 656,53$ \\
\hline
\end{tabular}

Os resultados obtidos via WJM_otimiza para a viga com carga de $10 \mathrm{kN} / \mathrm{m}$ apresentaram uma diferença de 5,53 \% em relação aos resultados obtidos em Klein (2008). Para a viga com carga de $20 \mathrm{kN} / \mathrm{m}$ os valores apresentaram diferença de 6,54 \% em relação aos resultados obtidos em Klein (2008). Dessa maneira, percebe-se que os valores fornecidos pelo WJM_otimiza estão minimizando o custo do elemento estrutural estudado de forma satisfatória, respeitando a norma de dimensionamento de estruturas de concreto armado NBR 6118 (ABNT, 2014).

\subsection{Aplicações de acordo com o plano de análise}

Os resultados das aplicações podem ser divididos em dois grupos. Para o primeiro grupo, percebe-se um resultado impróprio devido aos domínios de deformação. É possível visualizar que a restrição relativa à altura não foi respeitada em todo o processo (Ver maiores detalhes nos Quadros 3 e 4).

Nesse caso, avalia-se que os métodos determinísticos de Newton e Penalidade Exterior (MPE) possuem algumas desvantagens que podem estar causando essa inconsistência numérica. Uma delas é que a derivada segunda da função pseudo-objetivo não é contínua sobre a fronteira que limita a região viável. Como se fixou um valor para $\beta_{x}=0,45$, entende-se que o valor das variáveis para problemas com cargas pequenas, se mantém próximo à fronteira do problema, como o dmin para essa situação é muito menor que a restrição de desigualdade $(h \leq 30 \mathrm{~cm})$, a solução não estaria nessa fronteira. Segundo Mota (2010), a dificuldade com a penalidade exterior pode ser evitada utilizando métodos de penalidades mais refinados como o lagrangeano aumentado.

Quadro 3: Resultados gerais para carga de $2 \mathrm{kN} / \mathrm{m}$.

\begin{tabular}{|c|c|c|c|c|c|c|c|}
\hline $\mathbf{L}(\mathbf{c m})$ & $\mathbf{d} \mathbf{m i n}(\mathbf{c m})$ & $\mathbf{h}(\mathbf{c m})$ & $\mathbf{A s}\left(\mathbf{c m}^{\mathbf{2}}\right)$ & $\mathbf{C F}(\mathbf{R} \mathbf{)}$ & $\mathbf{C V}(\mathbf{R} \mathbf{)}$ & $\mathbf{C A}(\mathbf{R} \mathbf{)})$ & $\mathbf{C T}(\mathbf{R} \mathbf{)})$ \\
\hline 400,00 & 9,46 & 10,86 & 1,67 & 45,63 & 17,43 & 23,57 & 86,63 \\
\hline 450,00 & 10,65 & 12,23 & 1,87 & 55,26 & 22,07 & 29,97 & 107,30 \\
\hline
\end{tabular}




\begin{tabular}{|l|l|l|l|l|l|l|l|}
500,00 & 11,83 & 13,57 & 2,07 & 65,70 & 27,24 & 37,00 & 129,94 \\
\hline 550,00 & 13,01 & 14,94 & 2,29 & 77,06 & 32,97 & 45,03 & 155,06 \\
\hline
\end{tabular}

Quadro 4: Resultados gerais para carga de $5 \mathrm{kN} / \mathrm{m}$.

\begin{tabular}{|l|c|c|c|c|c|c|c|}
\hline $\mathbf{L}(\mathbf{c m})$ & $\mathbf{d} \mathbf{m i n}(\mathbf{c m})$ & $\mathbf{h}(\mathbf{c m})$ & $\mathbf{A s} \mathbf{( c m}^{\mathbf{2}}$ ) & $\mathbf{C F}(\mathbf{R} \mathbf{)}$ & $\mathbf{C V}(\mathbf{R} \mathbf{)}$ & $\mathbf{C A}(\mathbf{R} \mathbf{)}$ & $\mathbf{C T}(\mathbf{R} \mathbf{)})$ \\
\hline 400,00 & 14,96 & 17,17 & 2,63 & 61,74 & 27,56 & 38,42 & 127,72 \\
\hline 450,00 & 16,84 & 19,32 & 2,96 & 75,64 & 34,88 & 48,83 & 159,35 \\
\hline 500,00 & 18,71 & 21,47 & 3,29 & 90,89 & 43,07 & 60,47 & 194,43 \\
\hline 550,00 & 20,57 & 23,61 & 3,61 & 107,52 & 52,10 & 73,35 & 232,97 \\
\hline
\end{tabular}

Observou-se um aumento de $\mathrm{R} \$ 41,09$ no custo da viga de $400 \mathrm{~cm}$ de vão para o acréscimo de $3 \mathrm{kN} / \mathrm{m}$ de carregamento distribuído. Para a viga com o vão de $550 \mathrm{~cm}$ o acréscimo total foi de $\mathrm{R} \$ 77,91 \mathrm{kN} / \mathrm{m}$.

A área de aço para este primeiro grupo está adequada, no Quadro 5 compara-se a área encontrada com os valores máximos e mínimos determinados pela NBR 6118 (ABNT, 2014).

Quadro 5: Verificação da restrição de área de aço máxima e mínima para o primeiro grupo.

\begin{tabular}{|c|c|c|c|c|}
\hline $\begin{array}{c}\text { Carga } \boldsymbol{q} \\
(\mathbf{k N} / \mathbf{m})\end{array}$ & $\begin{array}{c}\text { Vão } \\
(\mathbf{c m})\end{array}$ & $\begin{array}{c}\text { As } \mathbf{m i n} \\
\left(\mathbf{c m}^{\mathbf{2}}\right)\end{array}$ & $\begin{array}{c}\text { As máx } \\
\left(\mathbf{c m}^{\mathbf{2}}\right)\end{array}$ & $\begin{array}{c}\text { As calculado } \\
\left(\mathbf{c m}^{\mathbf{2}} \mathbf{)}\right.\end{array}$ \\
\hline 2,00 & 400,00 & 0,22 & 6,08 & 1,67 \\
\hline 2,00 & 550,00 & 0,31 & 8,36 & 2,29 \\
\hline 5,00 & 400,00 & 0,36 & 9,61 & 2,63 \\
\hline 5,00 & 550,00 & 0,49 & 13,22 & 3,61 \\
\hline
\end{tabular}

O segundo grupo é composto pelas análises dos resultados com cargas maiores 10, 12, 15 e $20 \mathrm{kN} / \mathrm{m}$, que ainda apresentam alguns problemas em relação a variável altura $(x 1)$ como o descrito anteriormente, mas percebe-se que a variável $x 2$ tem valores dentro dos limites aceitáveis. Os Quadros 6 a 9 a seguir mostram os resultados obtidos para essas análises com variações de carga e vão.

As alturas desse segundo grupo apresentaram uma maior tendência de ficar dentro dos limites estabelecidos pelo processo de otimização, sendo então necessário que se aplique um fator de correção para as alturas adequadas obtidas, pois do ponto de vista construtivo, elas não são praticáveis, seria ideal que esses tamanhos fossem arredondados para valores múltiplos de cinco.

Para as vigas em questão a diferença entre o maior e menor carregamento (10 kN/m e 20 $\mathrm{kN} / \mathrm{m}$ ) foi de $\mathrm{R} \$ 68,80$ referente ao vão de $400 \mathrm{~cm}$. Para a o vão de $550 \mathrm{~cm}$ a diferença monetária entre os carregamentos indicados alcançou o valor de $\mathrm{R} \$ 220,27$.

Quadro 6: Resultados gerais para carga de $10 \mathrm{kN} / \mathrm{m}$.

\begin{tabular}{|c|c|c|c|c|c|c|c|}
\hline $\mathbf{L}(\mathbf{c m})$ & $\mathbf{d} \mathbf{m i n}(\mathbf{c m})$ & $\mathbf{h}(\mathbf{c m})$ & $\mathbf{A s}\left(\mathbf{c m}^{\mathbf{2}}\right)$ & $\mathbf{C F}(\mathbf{R} \mathbf{)}$ & $\mathbf{C V}(\mathbf{R} \mathbf{)}$ & $\mathbf{C A}(\mathbf{R} \mathbf{)})$ & $\mathbf{C T}(\mathbf{R} \mathbf{)})$ \\
\hline 400 & 21,16 & 24,28 & 3,71 & 79,91 & 38,97 & 56,17 & 175,05 \\
\hline 450 & 23,80 & 27,31 & 4,18 & 98,62 & 49,32 & 71,36 & 219,30 \\
\hline 500 & 26,44 & 30,35 & 4,65 & 119,27 & 60,89 & 88,38 & 268,54 \\
\hline 550 & 29,10 & 33,39 & 5,10 & 128,96 & 66,98 & 98,29 & 294,23 \\
\hline
\end{tabular}

Quadro 7: Resultados gerais para carga de $12 \mathrm{kN} / \mathrm{m}$.

\begin{tabular}{|c|c|c|c|c|c|c|c|}
\hline $\mathbf{L}(\mathbf{c m})$ & $\mathbf{d} \min (\mathbf{c m})$ & $\mathbf{h}(\mathbf{c m})$ & $\mathbf{A s}\left(\mathbf{c m}^{2}\right)$ & $\mathbf{C F}(\mathbf{R} \mathbf{)}$ & $\mathbf{C V}(\mathbf{R} \mathbf{)}$ & $\mathbf{C A}(\mathbf{R} \mathbf{)}$ & $\mathbf{C T}(\mathbf{R} \mathbf{)})$ \\
\hline 400,00 & 23,18 & 26,60 & 4,07 & 85,83 & 42,69 & 62,19 & 190,71 \\
\hline
\end{tabular}




\begin{tabular}{|l|c|c|c|c|c|c|c|}
450,00 & 26,09 & 29,93 & 4,58 & 106,12 & 54,03 & 79,02 & 239,17 \\
\hline 500,00 & 28,99 & 33,25 & 5,09 & 128,53 & 66,70 & 97,84 & 293,07 \\
\hline 550,00 & 31,87 & 36,58 & 5,60 & 153,07 & 80,72 & 118,69 & 352,48 \\
\hline
\end{tabular}

Quadro 8: Resultados gerais para carga de $15 \mathrm{kN} / \mathrm{m}$.

\begin{tabular}{|c|c|c|c|c|c|c|c|}
\hline $\mathbf{L}(\mathbf{c m})$ & $\mathbf{d} \boldsymbol{\operatorname { m i n }} \mathbf{( c m})$ & $\mathbf{h}(\mathbf{c m})$ & $\mathbf{A s} \mathbf{( c m}^{\mathbf{2}} \mathbf{)}$ & $\mathbf{C F}(\mathbf{R} \mathbf{)}$ & $\mathbf{C V}(\mathbf{R} \mathbf{)}$ & $\mathbf{C A}(\mathbf{R} \mathbf{)}$ & $\mathbf{C T}(\mathbf{R} \mathbf{)})$ \\
\hline 400,00 & 25,92 & 29,74 & 4,55 & 93,85 & 47,73 & 70,53 & 212,11 \\
\hline 450,00 & 29,16 & 33,46 & 5,12 & 116,27 & 60,41 & 89,60 & 266,28 \\
\hline 500,00 & 32,39 & 37,18 & 5,69 & 141,06 & 74,58 & 110,96 & 326,60 \\
\hline 550,00 & 35,64 & 40,9 & 6,26 & 168,22 & 90,24 & 134,58 & 393,04 \\
\hline
\end{tabular}

Quadro 9: Resultados gerais para carga de $20 \mathrm{kN} / \mathrm{m}$.

\begin{tabular}{|l|c|c|c|c|c|c|c|}
\hline $\mathbf{L}(\mathbf{c m})$ & $\mathbf{d} \boldsymbol{\operatorname { m i n }} \mathbf{( \mathbf { c m } )}$ & $\mathbf{h}(\mathbf{c m})$ & $\left.\mathbf{A s} \mathbf{( c m}^{\mathbf{2}}\right)$ & $\mathbf{C F}(\mathbf{R} \mathbf{)}$ & $\mathbf{C V}(\mathbf{R} \mathbf{)}$ & $\mathbf{C A}(\mathbf{R} \mathbf{)}$ & $\mathbf{C T}(\mathbf{R} \mathbf{)})$ \\
\hline 400,00 & 29,93 & 34,34 & 5,26 & 105,61 & 55,11 & 83,13 & 243,85 \\
\hline 450,00 & 33,67 & 38,63 & 5,91 & 131,15 & 69,76 & 105,60 & 306,51 \\
\hline 500,00 & 37,42 & 42,93 & 6,57 & 159,42 & 86,11 & 130,75 & 376,28 \\
\hline 550,00 & 41,15 & 47,22 & 7,23 & 190,45 & 104,2 & 158,59 & 453,24 \\
\hline
\end{tabular}

A área de aço para este segundo grupo também está adequada. O Quadro 10 compara a área de aço do menor e maior vão, submetidas à menor e maior carga.

Quadro 10: Verificação da restrição de área de aço máxima e mínima para o segundo grupo

\begin{tabular}{|c|c|c|c|c|}
\hline $\begin{array}{c}\text { Carga q } \\
(\mathbf{k N} / \mathbf{m})\end{array}$ & $\begin{array}{c}\text { Vão } \\
(\mathbf{c m})\end{array}$ & $\begin{array}{c}\text { As } \mathbf{m i n} \\
\left(\mathbf{c m}^{\mathbf{2}}\right)\end{array}$ & $\begin{array}{c}\text { As máx } \\
\left(\mathbf{c m}^{\mathbf{2}}\right)\end{array}$ & $\begin{array}{c}\text { As calculado } \\
\left(\mathbf{c m}^{\mathbf{2}}\right)\end{array}$ \\
\hline 10 & 400,00 & 0,50 & 13,59 & 3,71 \\
\hline 10 & 550,00 & 0,70 & 18,69 & 5,10 \\
\hline 20 & 400,00 & 0,72 & 19,23 & 5,26 \\
\hline 20 & 550,00 & 0,99 & 26,44 & 7,23 \\
\hline
\end{tabular}

Para verificar quanto cada material contribui para o custo final da viga, observa-se a Tabela 2 a seguir com a relação do custo média de cada material em relação ao custo total da viga analisada.

Percebe-se que o custo percentual com a forma tende a diminuir e o custo do volume de concreto e da área de aço tende aumentar, fato que ocorre devido ao aumento progressivo do momento fletor aplicado e de que a variável de projeto x1 (altura) é bastante sensível ao processo de otimização realizado.

Para perceber a interferência do vão em cada material, observa-se a Tabela 3 com a relação dos custos dos materiais e o custo total em função do vão da viga analisado.

Tabela 2: Relação percentual entre o custo dos materiais e o custo total da viga em função da carga

\begin{tabular}{c|c|c|c}
\hline $\begin{array}{c}\text { Carga q } \\
\text { (kN/m) }\end{array}$ & $\begin{array}{c}\text { CF } \\
\text { (\%) }\end{array}$ & $\begin{array}{c}\text { CV } \\
\text { (\%) }\end{array}$ & $\begin{array}{c}\text { CA } \\
\text { (\%) }\end{array}$ \\
\hline 2,00 & 51,11 & 20,73 & 28,16 \\
5,00 & 47,18 & 22,00 & 30,83 \\
10,00 & 44,72 & 22,55 & 32,74 \\
12,00 & 44,16 & 22,66 & 33,18 \\
15,00 & 43,48 & 22,75 & 33,87 \\
20,00 & 42,62 & 22,81 & 34,57 \\
\hline
\end{tabular}


Tabela 3: Relação porcentual média entre o custo dos materiais e o custo total da viga em função do vão

\begin{tabular}{c|c|c|c}
\hline $\begin{array}{c}\text { Vão } \\
\text { (cm) }\end{array}$ & $\begin{array}{c}\text { CF } \\
\text { (\%) }\end{array}$ & $\begin{array}{c}\text { CV } \\
\text { (\%) }\end{array}$ & $\begin{array}{c}\text { CA } \\
\text { (\%) }\end{array}$ \\
\hline 400,00 & $46,54 \%$ & $21,91 \%$ & $31,55 \%$ \\
450,00 & $45,79 \%$ & $22,16 \%$ & $32,04 \%$ \\
500,00 & $45,19 \%$ & $22,38 \%$ & $32,43 \%$ \\
550,00 & $44,65 \%$ & $22,54 \%$ & $32,81 \%$ \\
\hline
\end{tabular}

Dessa forma, como acontece com a situação de aumento de carregamento, o aumento do vão também acarreta um aumento no momento fletor, fazendo com que a altura seja a variável mais sensível no processo de otimização.

Ambos os quadros de relação percentual demonstram que o custo com aço é proporcionalmente maior que o custo do volume de concreto, e a diferença desse percentual é maior para cargas e vãos maiores, o que já era esperado para uma viga retangular de armadura simples.

Com todos os resultados obtidos, elaborou-se ábacos, com a altura, área de aço e o custo total das vigas, todos em função do vão livre da viga. Os mesmos são apresentados na Figura 5.

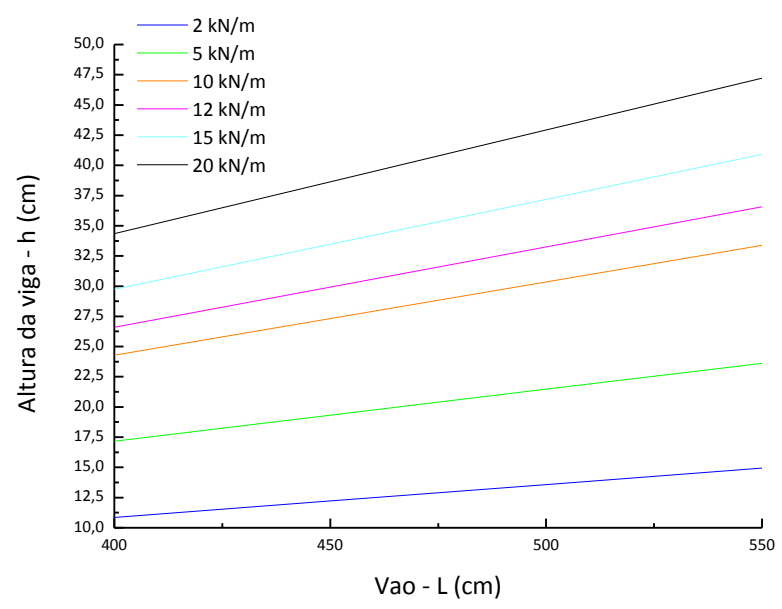

(a)

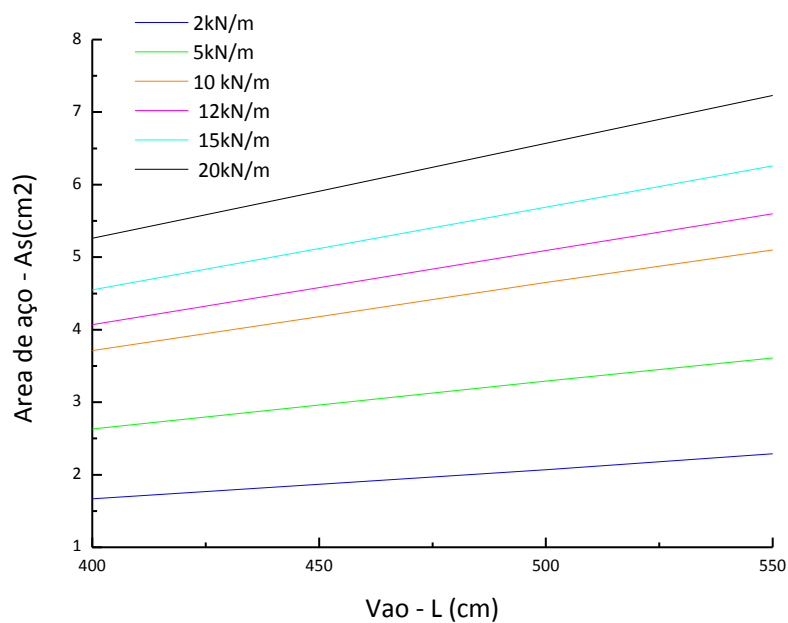

(b) 


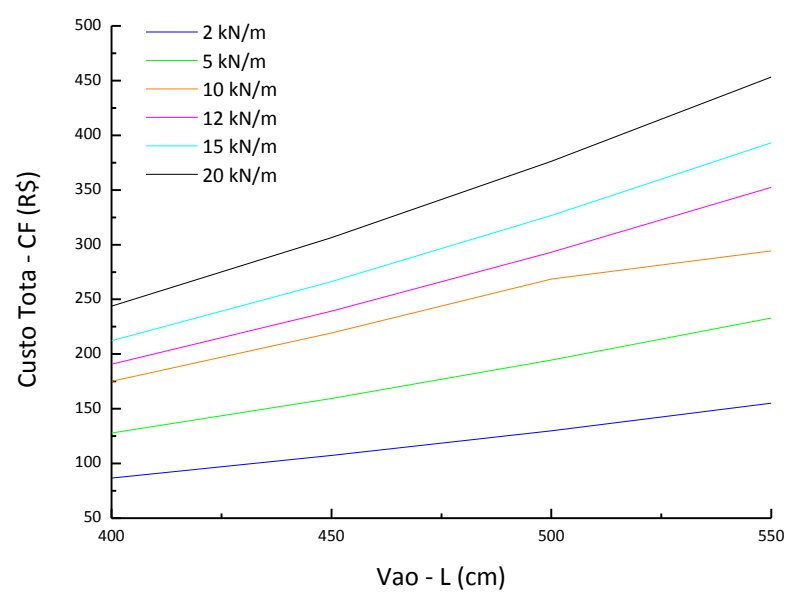

(c)

Figura 5: Ábaco vão livre $(\mathrm{cm})$ vs. altura da viga $(\mathrm{cm})(\mathrm{a})$; Ábaco vão livre $(\mathrm{cm})$ vs. área de aço $\left(\mathrm{cm}^{2}\right)(\mathrm{b})$ Ábaco vão livre $(\mathrm{cm})$ vs. custo da viga ( $\mathrm{R} \$$ ).

De acordo com os ábacos, nota-se o comportamento linear da altura, área de aço e do custo da viga em relação ao vão livre, fato que é comprovado pela descrição da FO, que é uma função linear.

\section{CONCLUSÃO}

Foi verificado que a rotina chamada WJM_otimiza teve sua validade comprovada através da comparação dos resultados obtidos com os Klein (2008). Outro fator importante é o fato de que as variáveis de projeto e custo obtidos dos exemplos numéricos alcançaram resultados reais para o problema estudado.

Mesmo que apresentando, em certos casos, valores fora dos padrões indicados em projeto, o processo de otimização pode ser uma ferramenta útil para determinar os valores das variáveis ótimas do projeto, podendo gerar economia, velocidade na elaboração em projetos, sempre atendendo os critérios normativos impostos pela NBR 6118 (ABNT, 2014).

Percebe-se que os resultados levaram ao maior aproveitamento da armadura e uma significativa redução da altura na seção transversal, isso implica na redução dos custos da forma, sendo este o responsável por mais de $40 \%$ dos custos nas peças analisadas.

No caso deste trabalho, foi utilizada uma abordagem clássica que conduziu a resultados coerentes do ponto de vista estrutural, portanto, outras abordagens podem ser utilizadas a fim de verificar métodos, por exemplo, de natureza heurística.

Como sugestão de trabalhos futuros especifica-se: (a) o teste de outras seções transversais, como a seção "T", seção "I" e seção "U"; (b) A utilização de metodologias de natureza bioinspirado, como o algoritmo de colônia de vagalumes e algoritmos genéticos; (c) verificar a sensibilidade dos parâmetros de entrada através de um estudo do desempenho computacional da rotina; (d) Realizar mais análises de forma a elaborar ábacos que possam auxiliar o pré-dimensionamento de vigas de concreto submetida a flexão; e (e) Avaliar a peça na condição de um dimensionamento para cisalhamento e verificações em Estado Limite de Serviço (ELS). 


\section{REFERÊNCIAS}

ABBAS, S. (2017). Golden Ratio: A Measure of Physical Beauty. Resonance, 22 (1), 51-60.

ABNT NBR 6118, de 07 de agosto de 2014 Projeto de estruturas de concreto - procedimento. São Paulo: Associação Brasileira de Normas Técnicas.

BASTOS, P. S. (2015). Flexão Normal Simples. Departamento de Engenharia Civil, Universidade Estadual Paulista, Bauru, São Paulo, Brasil.

BRAUN, E. (2016). Aplicação de métodos de otimização no dimensionamento de vigas de aço (Trabalho de Conclusão de curso em Engenharia Civil). Departamento de Engenharia Civil, UFRGS, Porto Alegre, Brasil.

HERNÁNDEZ, S.; FLORES, I.; VÁZQUEZ, J. (2012). A. Improved Golden-Section Algorithm for the Multi-Item Replenishment Problem. Journal of Applied Research and Technology, 10 (3), 388397.

KLEIN, H. W. (2008). Otimização de vigas de concreto armado: Dimensionamento a flexão (Trabalho de Conclusão de curso em Engenharia Civil). Departamento de Tecnologia, Universidade Regional do Noroeste do Estado do Rio Grande do Sul, Rio Grande do Sul, Brasil.

LEPS, M.; SEJNOHA, M. (2003). New approach to optimization of reinforced concrete beams. Computers and Structures, 81, 1957-1966.

LIU, S.; AN, X.; JIA, H. (2007). Topology optimization of beam cross-section considering warping deformation. Structural and Multidisciplinary Optimization, 35 (5), 403-411.

MAIA, J. P. (2009). Otimização estrutural: estudo e aplicações em problemas clássicos de vigas utilizando a ferramenta Solver. (Dissertação de mestrado em Engenharia Civil - Estrutura). Escola de Engenharia de São Carlos, Universidade Federal de São Carlos, São Carlos, São Paulo, Brasil.

MOTA, A. М. T. (2010). Um metodo de redução para programação semi-infinita não linear baseado numa tecnica de penalidade exacta. (Tese Doutorado em Engenharia Industrial e de Sistemas). Escola de Engenharia, Universidade do Minho, Braga, Portugal.

MUÑIZ JUNIOR, F. H. M. M.; OLIVEIRA, D. M. (2014). Otimização de vigas de concreto armado com seção retangular submetidas à flexão normal simples. Construindo, 6 (1), 51-57.

SEN, S. K.; AGARWAL, R. P. (2007). Golden ratio in science, as random sequence source, its computation and beyond. Computers and Mathematics with Applications, 56, 469-498.

SILVA, E. C. (20--). Otimização aplicada ao projeto de sistemas mecânicos. Escola Politécina da USP, Departamento de Engenharia Mecatrônica e Sistemas Mecânicos, Universidade de São Paulo, São Paulo, São Paulo, Brasil.

VANDERPLAATS, G. (1999). Numerical Optimization Techiniques for Engineering Design (3a ed.). Colorado Springs: McGraw-Hill Ryerson. 
VANDERPLAATS, G. (2006). Structural Optimization for Statics, Dynamics and Beyond. Journal of the Brazilian Society of Mechanical Sciences and Engineering, 28 (3), 316-322.

VASCONCELOS, R. F., ALMEIDA, S. R. M.; PRADO, A. A. (2013, Novembro). Otimização de lajes alveolares pré-moldadas de concreto protendido segundo as normas brasileiras. Proceedings of the XXXIV Iberian Latin-American Congress on Computational Methods in Engineering. Pirenópolis. CD-ROM.

VASCONCELOS, R. F. (2014). Otimização de elementos pré-moldados de concreto: lajes alveolares e vigas com cabo reto. (Dissertação de Mestrado em Engenharia Civil - Estrutura). Escola de Engenharia Civil e Ambiental, Universidade Federal de Goiás, Goiânia, Goiás, Brasil.

VENKATARAMAN, P. (2001). Applied Optimization with Matlab programming (3a ed.), Nova lorque: John Wiley \& Sons. 